\title{
1
}

\section{Déficit agroalimentario de la provincia de Santa Elena}

Bruno Burgos Vera, Otto Suárez Rodríguez 


\title{
Déficit agroalimentario de la provincia de Santa Elena Agri-food deficit of Santa Elena Province
}

\author{
Bruno Burgos Vera; Otto Suárez Rodríguez \\ Universidad Tecnológica Equinoccial, Santa Elena - Ecuador \\ Correo electrónico: bburgosvera@ @otmail.com
}

\begin{abstract}
Resumen
El desarrollo de una nación se cimienta principalmente en el bienestar de su colectivo a través de una redistribución equitativa del ingreso, una mayor productividad y un incremento de las oportunidades laborales; la agricultura juega como tal un rol fundamental para la economía y nutrición del colectivo. Sin embargo, las políticas de estado se han orientado generalmente hacia otros sectores como la industria, comercio y minería. Si a esto se agrega las inadecuadas inversiones en investigación científica, capacitación y transferencia de recursos y tecnología, se tiene como resultado una baja productividad y limitado aprovechamiento de los recursos que ofrece la agricultura poniendo en riesgo la soberanía alimentaria del país, objetivo estratégico fundamental consagrado en la Constitución. Esta situación es más crítica a nivel provincial, en que provincias como Santa Elena no cuentan con los recursos adecuados y suficientes para crear una suficiencia agroalimentaria sostenible a largo plazo. Este estudio, en base a información secundaria, analiza la situación actual de la producción agropecuaria en la provincia de Santa Elena con el fin de inferir sobre la suficiencia agroalimentaria de su población y los retos que enfrenta la provincia para lograr una soberanía alimentaria que tienda las bases de su desarrollo.
\end{abstract}

Palabras Clave: Soberanía alimentaria, suficiencia agroalimentaria, producción agropecuaria, Santa Elena

\begin{abstract}
The development of a country is mainly based on the welfare of its people through a fair redistribution of the income, a higher productivity, and the increase of work opportunities; as such the agricultural sector plays a key role for the economy and nutrition of the people. However, public policies have usually aimed at other sectors such as industry, commerce, and mining. Moreover, poor investments in scientific research, training and transfer of technology and resources contribute to low productivity and limited use of the products offered by agriculture, putting at risk the food sovereignty of the country, a key strategic objective recognized by the Constitution. This situation is even more critical to the provincial level, where provinces, such as Santa Elena, do not have enough and adequate resources to be agri-food self-sufficient and sustainable in the long run. The present study, based on secondary information, analyzes the current situation of agricultural production in the Province of Santa Elena in order to infer about the food sufficiency of its people and the challenges ahead for this province in its quest to reach food sovereignty and a steady development.
\end{abstract}

Key Words: Food sovereignty, agri-food sufficiency, agricultural production, Santa Elena Province 


\section{Introducción}

El Ecuador presenta una diversidad de espacios geográficos y ecosistemas, así como una gran variedad de pisos climáticos y condiciones geomorfológicas que configuran una potencialidad agrícola limitada, salvo contados casos como la cuenca del río Guayas y algunos valles interandinos. Si se considera además que una gran parte de la producción agrícola ecuatoriana se destina para la exportación (banano, cacao, café, entre otros productos), la producción agrícola destinada a la alimentación de los ecuatorianos es aún más limitada, lo cual tendría serias repercusiones sobre la disponibilidad de alimentos adecuados para la buena nutrición de los ecuatorianos.

En 1986, la encuesta nacional sobre la Situación Alimentaria, Nutricional y de Salud de la Población de Niños Ecuatorianos menores de cinco años (DANS) reveló la existencia de elevadas tasas de desnutrición aguda, desnutrición global y desnutrición crónica o retardo en talla. También reveló la existencia de deficiencias específicas de micronutrientes, en particular hierro y zinc; además, mostró una enorme desigualdad de las condiciones de salud y nutrición en niños entre la población urbana y rural y entre la sierra y la costa [4].

Posterior a la DANS, en el 2012, el Ministerio de Salud estimó prevalencias de anemias superiores a las encontradas en 1986 en la población de escasos recursos económicos, además se demostró que el retardo en talla, así como la desnutrición siguen siendo prevalentes en el país, a lo cual se han sumado problemas recientes como el sobrepeso y la obesidad resultantes de la malnutrición y los malos hábitos alimenticios [4]. La malnutrición es un trastorno fisiológico causado por una ingesta insuficiente, excesiva o desequilibrada de macronutrientes (hidratos de carbono, proteínas, lípidos) y de micronutrientes (vitaminas y minerales). Es en sí una nutrición deficiente que ocasiona problemas de salud por comer muy poco (subnutrición), demasiado (hipernutrición) o comer en forma desequilibrada en cuanto al balance de nutrientes esenciales para una vida sana [2].

Si bien no existe en el país un estudio que demuestre fehacientemente cómo los ecuatorianos se nutren en cantidad (energía) y calidad (diversidad de nutrientes) de los alimentos que provienen de la producción agropecuaria del país, se podría prever que ésta es insuficiente para alimentar adecuadamente a los ecuatorianos, sin contar obviamente el acceso limitado de una gran parte de la población a alimentos en cantidad y calidad adecuadas por su reducido poder adquisitivo.

Este argumento se mantiene en el caso de la provincia de Santa Elena, donde la situación agroproductiva tiende a ser más crítica que en otros sectores del país, por sus características edafológicas propias y el poco acceso al agua que no han permitido el desarrollo agropecuario de la península de Santa Elena.

\section{Antecedentes \\ Soberanía alimentaria}

El ser humano desde sus inicios ha tenido como premisa básica el satisfacer sus necesidades, deseos y aspiraciones, procurando siempre la supervivencia de la especie. Uno de los aspectos claves para la supervivencia es la alimentación que permite al individuo crecer de una manera saludable y mantener el capital humano en óptimas condiciones, de manera que la sociedad cuente permanentemente con individuos sanos y productivos que contribuyan al desarrollo sostenido y la perpetuidad de la especie humana. Así mismo, desde sus inicios el ser humano se procuró sus alimentos de la tierra y el mar y esto sigue siendo tan cierto como en aquellos primeros tiempos. Sin embargo, las condiciones actuales de la producción agropecuaria no permiten alimentar en forma adecuada a la población mundial, múltiples son las causas de esta situación, lo que escapa al presente estudio, pero allí radica el origen del concepto de soberanía alimentaria en boga desde 1996 como respuesta al ineficiente sistema alimentario mundial, que no ha sido capaz de cumplir con su función principal: alimentar a las personas.

La Declaración de Nyéléni durante el Foro para la Soberanía Alimentaria celebrado en Mali en el 2007 [3] definió a la soberanía alimentaria como "el derecho de los pueblos a alimentos nutritivos y culturalmente adecuados, accesibles, producidos de forma sostenible y ecológica, y su derecho a decidir su propio sistema alimentario y productivo".

La Constitución de la República del Ecuador [1] en su Artículo 281 estipula que "la soberanía alimentaria constituye un objetivo estratégico y una obligación del Estado para garantizar que las personas, comunidades, pueblos y nacionalidades alcancen la autosuficiencia de alimentos sanos y culturalmente apropiados de forma permanente".

\section{La provincia de Santa Elena}

El 7 de noviembre del 2007 se creó la provincia de Santa Elena como unidad territorial, política y administrativa, integrada por los cantones Santa Elena, Salinas y La Libertad, manteniendo los límites territoriales que poseían cuando eran parte de la provincia del Guayas. 
Más allá de las motivaciones esgrimidas para la creación de la provincia, entre las que figuraba la identidad cultural peninsular y su historia, Santa Elena empezó dando sus primeros pasos sin una base productiva sólida y con una débil infraestructura particularmente en el área rural. La economía de la provincia está basada en el turismo y la pesca. La pesca es principalmente artesanal y se centra en sus principales puertos pesqueros Santa Rosa, Anconcito y Chanduy. El comercio se caracteriza por el intercambio de productos terminados (consumo final) e insumos para algunas actividades semi-industriales, artesanales, entre otras. La administración es descentralizada; es decir, los procesos políticosadministrativos se los maneja con independencia del gobierno central, salvo los considerados estratégicos como el petróleo.

La dinámica económica generada por el turismo, que incluye los servicios de hospedaje, alimentación, recreación y entretenimiento, los servicios financieros, las cadenas de provisión de productos de consumo masivo e insumos $y$ algunas actividades relacionadas con la acuacultura y la pesca, se sostiene en inversiones que en su mayoría provienen de ciudades como Guayaquil y Quito o incluso de fuera del país. Esto significa que los réditos de esta gestión, no se quedan o reinvierten en la provincia, incluso los tributos que se generan, nutren las arcas de los lugares donde estas empresas tiene su origen o base jurídica, lo que atenta al desarrollo de la provincia y contribuye para que ésta se vuelva dependiente en extremo de las asignaciones que provienen del gobierno central. Las pocas fuentes propias de inversión se dan en sectores como la pesca artesanal, algunas actividades turísticas y de comercio local y en menor escala de la agricultura.
La provincia de Santa Elena tiene una población de 358.896 habitantes (2,20\% del total nacional), según la proyección al 2015 del INEC [5]; la misma que se encuentra concentrada en las tres cabeceras cantonales (Santa Elena, La Libertad y Salinas) en un $90 \%$; el $10 \%$ restante se encuentra repartida entre las parroquias rurales de la provincia.

La superficie provincial equivale al $1,46 \%$ del total nacional, es decir, $3.762,8 \mathrm{~km}^{2}$. Dado que la población se concentra en un $90 \%$ en $500 \mathrm{~km}^{2}$ de superficie que corresponde a las cabeceras cantonales, la densidad poblacional urbana promedio es de 646 habitantes por $\mathrm{km}^{2}$ versus 11 habitantes por $\mathrm{km}^{2}$ en el área rural.

\section{Diagnóstico situacional y análisis Uso agrícola del suelo}

Según el INEC - ESPAC [6], al 2015 la provincia de Santa Elena contaba con una superficie de 79.525 hectáreas para uso agrícola del suelo; es decir, sólo el 21,10\% del total de su superficie (376.280 hectáreas) está destinado a las actividades agropecuarias.

$\mathrm{Si}$ se compara a nivel nacional con la superficie destinada a las labores agrícolas y pecuarias (12`585.861 hectáreas) que corresponde al $48,83 \%$ del total de la superficie del país, la provincia de Santa Elena está muy por debajo de este promedio nacional, lo que en parte se explica por las características seca y semidesértica de esta región del país.

La Tabla 1 presenta la distribución del suelo de la provincia de Santa Elena entre sus diferentes usos agrícolas durante los últimos 7 años.

Tabla 1. Uso agrícola del suelo de la provincia de Santa Elena (superficie en hectáreas)

\begin{tabular}{|c|c|c|c|c|c|c|c|c|}
\hline Año & Total & $\begin{array}{c}\text { Cultivos } \\
\text { Permanentes }\end{array}$ & \begin{tabular}{l}
\multicolumn{1}{c}{ Cultivos } \\
Transitorios \\
y Barbecho
\end{tabular} & Descanso & $\begin{array}{c}\text { Pastos } \\
\text { Cultivados }\end{array}$ & $\begin{array}{c}\text { Pastos } \\
\text { Naturales }\end{array}$ & $\begin{array}{c}\text { Montes } \\
\text { y Bosques }\end{array}$ & $\begin{array}{l}\text { Otros } \\
\text { Usos }\end{array}$ \\
\hline \multirow{2}{*}{2009} & \multirow{2}{*}{138.565} & 2.119 & 7.076 & 3.447 & 10.517 & 32.877 & 79.750 & 2.780 \\
\hline & & $1,53 \%$ & $5,11 \%$ & $2,49 \%$ & $7,59 \%$ & $23,73 \%$ & $57,55 \%$ & $2,01 \%$ \\
\hline \multirow{2}{*}{2010} & \multirow{2}{*}{135.201} & 2.608 & 4.477 & 2.554 & 11.962 & 31.182 & 78.000 & 4.418 \\
\hline & & $1,93 \%$ & $3,31 \%$ & $1,89 \%$ & $8,85 \%$ & $23,06 \%$ & $57,69 \%$ & $3,27 \%$ \\
\hline \multirow{2}{*}{2011} & \multirow{2}{*}{127.094} & 3.659 & 8.982 & 2.171 & 11.908 & 2.579 & 90.685 & 7.109 \\
\hline & & $2,88 \%$ & $7,07 \%$ & $1,71 \%$ & $9,37 \%$ & $2,03 \%$ & $71,35 \%$ & $5,59 \%$ \\
\hline \multirow{2}{*}{2012} & \multirow{2}{*}{128.351} & 3.387 & 8.371 & 2.634 & 12.759 & 14.411 & 80.404 & 6.386 \\
\hline & & $2,64 \%$ & $6,52 \%$ & $2,05 \%$ & $9,94 \%$ & $11,23 \%$ & $62,64 \%$ & $4,98 \%$ \\
\hline \multirow{2}{*}{2013} & \multirow{2}{*}{129.920} & 3.358 & 11.572 & 4.597 & 20.713 & 20.511 & 62.115 & 7.055 \\
\hline & & $2,58 \%$ & $8,91 \%$ & $3,54 \%$ & $15,94 \%$ & $15,79 \%$ & $47,81 \%$ & $5,43 \%$ \\
\hline \multirow[b]{2}{*}{2014} & \multirow{2}{*}{76.495} & 5.475 & 6.039 & 4.813 & 7.105 & 1.961 & 48.717 & 2.385 \\
\hline & & $7,16 \%$ & $7,89 \%$ & $6,29 \%$ & $9,29 \%$ & $2,56 \%$ & $63,69 \%$ & $3,12 \%$ \\
\hline \multirow{2}{*}{2015} & \multirow{2}{*}{79.525} & 3.101 & 5.026 & 2.228 & 6.352 & 2.217 & 56.940 & 3.661 \\
\hline & & $3,90 \%$ & $6,32 \%$ & $2,80 \%$ & $7,99 \%$ & $2,79 \%$ & $71,60 \%$ & $4,60 \%$ \\
\hline
\end{tabular}

Fuente: INEC - Elaboración: autores 
Cabe destacar la significativa reducción de la frontera agrícola a lo largo de estos años (2009 2015), de 138.565 a 79.525 hectáreas, lo que representa una reducción del 42,6\%; sin embargo, sólo entre el 2013 y el 2014 se redujo $41,1 \%$ lo que se explica principalmente por la disminución del área de pastos naturales y cultivados.

Las áreas dedicadas a cultivos han promediado un $10 \%$ del total de uso agrícola del suelo durante el período, aunque con un incremento paulatino en los últimos años, llegando al $15 \%$ en el 2014 , éstas son en sí las áreas donde se producen los alimentos de origen agrícola a las que se deben sumar las áreas de pastos de donde salen los alimentos de origen pecuario, que en el 2015 correspondieron a casi el $11 \%$ del total. Es decir, del total del área de uso agrícola de la provincia, apenas un $21 \%$ se destina a la producción de alimentos de origen agrícola y pecuario.

\section{Producción agrícola}

Según las estadísticas del INEC - ESPAC [6], en el 2015 los cultivos relevantes entre permanentes y transitorios de la provincia fueron: banano, cacao, café, maracuyá, plátano, maíz duro y soya; cuyas superficies, producción y rendimiento se presentan en la Tabla 2.

Tabla 2. Superficie, producción y rendimientos de principales cultivos en la provincia de Santa Elena (año 2015)

\begin{tabular}{|c|c|c|c|c|c|}
\hline \multirow{2}{*}{ Cultivo } & \multicolumn{2}{|c|}{$\begin{array}{l}\text { Superficie } \\
\text { (hectáreas) }\end{array}$} & \multirow{2}{*}{$\begin{array}{l}\text { Producción } \\
\text { (tm) }\end{array}$} & \multirow{2}{*}{$\begin{array}{c}\text { Rendimiento } \\
(\mathrm{tm} / \mathrm{ha})\end{array}$} & \multirow{2}{*}{$\begin{array}{c}\text { Rendimient } \\
\text { o promedio } \\
\text { nacional } \\
(\mathrm{tm} / \mathrm{ha})\end{array}$} \\
\hline & Plantada & Cosechada & & & \\
\hline Banano (fruta fresca) & $250^{*}$ & 250 & 4.870 & 19,48 & 38,79 \\
\hline Cacao (almendra seca) & $278^{*}$ & 266 & 176 & 0,66 & 0,42 \\
\hline Café (grano oro) & $95 * *$ & --- & --- & --- & --- \\
\hline Maracuyá (fruta fresca) & $424 *$ & 389 & 1.618 & 4,16 & 5,67 \\
\hline Plátano & $93^{*}$ & 56 & 378 & 6,75 & 6,38 \\
\hline Maíz duro choclo & $44^{*}$ & 44 & 233 & 5,30 & 0,92 \\
\hline Maíz duro seco & $3.544^{*}$ & 3.360 & 17.309 & 5,15 & \multirow{2}{*}{4,47} \\
\hline Maíz duro seco & $92 * *$ & 92 & 504 & 5,48 & \\
\hline Soya & $19 *$ & 19 & 26 & 1,37 & 1,45 \\
\hline
\end{tabular}

*Solo; **Asociado

Fuente: INEC

Elaboración: autores

Estos cultivos representan cerca del $60 \%$ de las áreas dedicadas a los cultivos permanentes y transitorios, la diferencia corresponde a un sinnúmero de cultivos menores y que no son representativos.

Se observa también que el maíz duro seco es el cultivo predominante en superficie y en producción pues corresponde al $75 \%$ y $71 \%$, respectivamente, siguiéndole en importancia en área sembrada el maracuyá, el cacao y el banano y en producción el banano y el maracuyá. Cabe indicar que el café no registra producción pues al parecer es un cultivo nuevo que aún no produce. Analizando los rendimientos, sólo el banano, el maracuyá y la soya tienen rendimientos por debajo del promedio nacional, los demás cultivos muestran rendimientos por encima del promedio nacional. Esto implicaría que existen esfuerzos importantes por tecnificar y manejar adecuadamente los cultivos, a pesar de las condiciones del suelo y climáticas que caracterizan esta provincia (escasa pluviosidad, ambiente salino, suelos arenosos), situaciones como las formas de tenencia de la tierra (comunas, reservas naturales) y la existencia de mano de obra no calificada. No obstante, esta importante observación, el principal esfuerzo de la provincia en el corto plazo debiera orientarse hacia una utilización más extensiva de su tierra de la mano con mejores tecnologías, acceso al agua y mayores inversiones agroindustriales. Mantener los niveles de rendimientos a los niveles actuales o incluso mayores es el verdadero desafío con una agricultura extensiva antes que lo logrado en las pequeñas extensiones que se manejan hasta ahora.

\section{Producción pecuaria}

En cuanto a la producción de ganado, Santa Elena muestra cifras que de una u otra forma se relacionan con su limitado uso del suelo. La Tabla 3 presenta la evolución de las diferentes especies de ganado en la provincia desde el 2009 al 2015. 
Tabla 3. Evolución del ganado por especie en la provincia de Santa Elena (en cabezas de ganado)

\begin{tabular}{|r|r|r|r|r|r|r|r|}
\hline Año & Vacuno & \multicolumn{1}{c|}{ Porcino } & \multicolumn{1}{c|}{ Ovino } & \multicolumn{1}{c|}{ Asnal } & \multicolumn{1}{c|}{ Caballar } & \multicolumn{1}{c|}{ Mular } & \multicolumn{1}{c|}{ Caprino } \\
\hline 2009 & 14.543 & 2.734 & 455 & 830 & 2.421 & 325 & 4.814 \\
\hline 2010 & 17.379 & 3.866 & 549 & 632 & 1.783 & 453 & 9.693 \\
\hline 2011 & 15.966 & 5.255 & 902 & 568 & 2.595 & 379 & 8.571 \\
\hline 2012 & 18.448 & 5.810 & 216 & 1.091 & 4.104 & 545 & 9.292 \\
\hline 2013 & 23.282 & 6.325 & 536 & 219 & 2.253 & 711 & 6.436 \\
\hline 2014 & 10.454 & 7.972 & 11.452 & 221 & 1.004 & 286 & 330 \\
\hline 2015 & 7.275 & 6.605 & 23 & 67 & 846 & 283 & 2.221 \\
\hline
\end{tabular}

Fuente: INEC

Elaboración: autores

La evolución dada entre los años 2009 y 2013, hasta cierto punto es aceptable pues responde a variaciones propias de un proceso de gestión de comercialización o intercambio natural; pero éste no es el caso en los años 2014 y 2015 que presentan cifras inusuales, especialmente en el ganado vacuno, ovino y caprino. Al respecto, la Tabla 4 confronta el promedio de los años 2009 - 2013 con las cifras del 2014 y 2015.

Tabla 4. Comparación de la existencia de ganado entre el período 2009 - 2013 con el 2014 y 2015

\begin{tabular}{|l|r|r|r|}
\hline \multirow{2}{*}{ Año } & \multicolumn{3}{c|}{ Ganado } \\
\cline { 2 - 4 } & \multicolumn{2}{|c|}{ Vacuno } & \multicolumn{1}{c|}{ Ovino } \\
\hline $\begin{array}{l}\text { Promedio } \\
(2009-2013)\end{array}$ & 17.924 & 532 & 7.761 \\
\hline 2014 & 10.454 & 11.452 & 330 \\
\hline Variación & $-41,68 \%$ & $+2.052,63 \%$ & $-95,75 \%$ \\
\hline 2015 & 7.275 & 23 & $-71,38 \%$ \\
\hline Variación & $-59,41 \%$ & $-95,68 \%$ & \\
\hline
\end{tabular}

Fuente: INEC

Elaboración: Los autores

Se observa que en el 2014 y 2015 el ganado vacuno ha tenido un decrecimiento promedio de aproximadamente $50 \%$ respecto del promedio de los cinco años anteriores; de otra parte, el crecimiento experimentado en el ganado ovino es fuera de lo común, pues de 532 cabezas en el quinquenio 2009 - 2013 aumentaron a 11.452 cabezas en el 2014, esto es más del $2.000 \%$ y en el 2015 abruptamente disminuyeron a 23 cabezas; finalmente para el ganado caprino la situación es igual de inusual pues, de un promedio de 7.761 cabezas del 2009 al 2013 se redujeron a 330 cabezas en el 2014 y 2.221 cabezas en el 2015 , esto es un decrecimiento del $95,75 \%$ y $71,38 \%$ en su orden. ${ }^{1}$

Independiente de estas variabilidades, el sector ganadero de la península como proveedor de alimentos es relativamente muy pequeño, comparado con los promedios nacionales, especialmente el ganado vacuno que representa apenas el $0,18 \%$ del total nacional.

$\mathrm{Si}$ además, se toman en cuenta los pastos cultivados en la provincia (6.352 hectáreas), que representan tan sólo el 0,25\% del total nacional, se puede estimar una carga promedio de ganado vacuno de 1,15 cabezas/ha en la provincia versus 1,63 cabezas/ha a nivel nacional, lo cual implicaría que las áreas de pastos se están subutilizando. Estas cifras reflejan el poco potencial que tiene la ganadería como proveedora de alimentos en la península.

En efecto, si se considera la producción de leche, uno de los alimentos básicos obtenido del ganado vacuno, la Tabla 5 presenta la producción de este líquido en la provincia y su comparativo con provincias vecinas y a nivel nacional.

\footnotetext{
${ }^{1}$ Más allá de las cifras el INEC no proporciona explicaciones de estas variaciones, por otra parte, el MAGAP se sujeta a las cifras dadas por el INEC.
} 
Tabla 5. Vacas ordeñadas, producción y destino de la leche, cuadro comparativo (año 2015)

\begin{tabular}{|l|r|r|r|r|r|r|r|}
\cline { 5 - 8 } & $\begin{array}{c}\text { No. de } \\
\text { vacas } \\
\text { ordeñadas }\end{array}$ & \multirow{2}{*}{$\begin{array}{c}\text { Producción } \\
\text { (litros) }\end{array}$} & $\begin{array}{c}\text { Vendida } \\
\text { en } \\
\text { líquido }\end{array}$ & $\begin{array}{c}\text { Consumida } \\
\text { en el } \\
\text { terreno }\end{array}$ & $\begin{array}{c}\text { Alimentación } \\
\text { al balde }\end{array}$ & $\begin{array}{c}\text { Procesada } \\
\text { en el } \\
\text { terreno }\end{array}$ & $\begin{array}{l}\text { Otros } \\
\text { fines }\end{array}$ \\
\hline Nacional & 860.886 & 4.982 .370 & 3.575 .277 & 397.600 & 97.886 & 901.766 & 9.841 \\
\hline Guayas & 44.051 & 171.214 & 100.011 & 16.036 & 1.430 & 53.419 & 318 \\
\hline Manabí & 168.122 & 609.863 & 227.034 & 43.487 & 4.400 & 331.853 & 3.089 \\
\hline $\begin{array}{l}\text { Santa } \\
\text { Elena }\end{array}$ & 200 & 567 & 166 & 111 & & & \\
\hline
\end{tabular}

Fuente: INEC

Elaboración: Los autores

Como se puede apreciar, el número de vacas en ordeño y por tanto la producción de leche en la provincia son ínfimos comparado con la producción nacional y de las provincias vecinas, además comparando los rendimientos promedios se tiene que Santa Elena presenta un rendimiento promedio de 2,84 litros por vaca ordeñada, lo cual está muy por debajo del promedio nacional de 5,79 litros, Guayas 3,89 litros y Manabí 3,63 litros por ordeño.
Por otro lado, la poca leche que se produce principalmente es consumida y procesada en la finca, es decir muy poco va para la venta. Este sería otro indicador más del déficit agroalimentario en la península.

En cuanto a la producción avícola, las Tablas 6 y 7 presentan el número de aves criadas tanto en planteles avícolas como en el campo en Santa Elena, a nivel nacional y en las provincias vecinas.

Tabla 6. Número de aves criadas en planteles avícolas por especie, cuadro comparativo (año 2015)

\begin{tabular}{|l|r|r|r|r|r|r|}
\cline { 2 - 7 } \multicolumn{1}{c|}{} & \multicolumn{1}{c|}{ Total } & \multicolumn{1}{c|}{$\begin{array}{c}\text { Gallinas } \\
\text { ponedoras }\end{array}$} & $\begin{array}{c}\text { Gallinas } \\
\text { reproductoras }\end{array}$ & $\begin{array}{c}\text { Pollitos/as, } \\
\text { pollos/as }\end{array}$ & \multicolumn{1}{c|}{ Pavos } & \multicolumn{1}{c|}{ Otras } \\
\hline Nacional & 43.156 .323 & 9.999 .484 & 2.275 .578 & 30.394 .969 & 239.666 & 246.626 \\
\hline Guayas & 6.892 .662 & 44.000 & 197.535 & 6.651 .127 & --- & --- \\
\hline Manabí & 2.577 .518 & 1.166 .178 & 256.800 & 1.154 .540 & --- & --- \\
\hline Santa Elena & 224.000 & --- & --- & 182.000 & 42.000 & --- \\
\hline
\end{tabular}

Fuente: INEC

Elaboración: Los autores

La provincia mantiene criaderos de pollos para carne en cantidades poco relevantes comparado con las cifras a nivel nacional y de las provincias vecinas; la producción de pavos es en todo caso más relevante con un $17,5 \%$ del total nacional. No se especifica a qué mercado están destinadas estas producciones.

Tabla 7. Número de aves criadas en el campo por especie, cuadro comparativo (año 2015)

\begin{tabular}{|l|r|r|r|r|c|}
\cline { 2 - 6 } \multicolumn{1}{c|}{} & \multicolumn{1}{c|}{ Total } & Gallos y gallinas & Pollitos/as, pollos/as & \multicolumn{1}{c|}{ Patos } & \multicolumn{1}{c|}{ Pavos } \\
\hline Nacional & 5.981 .304 & 2.616 .788 & 2.865 .791 & 447.793 & 50.932 \\
\hline Guayas & 814.216 & 324.933 & 373.499 & 105.212 & 10.571 \\
\hline Manabí & 1.613 .843 & 603.462 & 873.986 & 124.395 & 11.999 \\
\hline Santa Elena & 5.499 & 2.196 & 2.445 & 724 & 133 \\
\hline
\end{tabular}

Fuente: INEC

Elaboración: Los autores

De igual manera, la cantidad de aves criadas en el campo en Santa Elena es comparativamente muy baja, lo cual es otra evidencia de la deficitaria producción alimenticia en la península.
Finalmente, en cuanto a la producción de huevos de gallina, la Tabla 8 presenta así mismo la producción comparativa de Santa Elena con respecto a la producción nacional y de las provincias vecinas. 
Tabla 8. Producción y destino de huevos de gallina, cuadro comparativo (año 2015 en miles)

\begin{tabular}{|c|c|c|c|c|c|c|c|c|}
\hline & \multicolumn{4}{|c|}{ Aves criadas en el campo } & \multicolumn{4}{|c|}{ Aves criadas en planteles avícolas } \\
\hline & \multirow[b]{2}{*}{$\begin{array}{c}\text { Producció } \\
\mathbf{n}\end{array}$} & \multicolumn{3}{|c|}{ Destino } & \multirow[b]{2}{*}{$\begin{array}{c}\text { Producció } \\
\mathbf{n}\end{array}$} & \multicolumn{3}{|c|}{ Destino } \\
\hline & & $\begin{array}{c}\text { Auto } \\
\text { Consum } \\
\text { o }\end{array}$ & $\begin{array}{c}\text { Venta } \\
\text { S }\end{array}$ & $\begin{array}{l}\text { Otro } \\
\mathbf{s}\end{array}$ & & $\begin{array}{c}\text { Auto } \\
\text { Consum } \\
\text { o }\end{array}$ & Ventas & Otros \\
\hline $\begin{array}{l}\text { Naciona } \\
1\end{array}$ & $3.529,2$ & $2.893,1$ & 335,0 & 301,1 & $60.803,8$ & 73,0 & $\begin{array}{r}55.474, \\
7\end{array}$ & $\begin{array}{r}5.256, \\
1\end{array}$ \\
\hline Guayas & 349,2 & 275,6 & 42,0 & 31,6 & $1.632,1$ & --- & $1.632,1$ & --- \\
\hline Manabí & 785,8 & 606,9 & 64,2 & 114,7 & $10.083,2$ & 3,7 & $9,979,3$ & 100,2 \\
\hline $\begin{array}{l}\text { Santa } \\
\text { Elena }\end{array}$ & 2,9 & 2,9 & --- & --- & --- & --- & --- & --- \\
\hline
\end{tabular}

Fuente: INEC

Elaboración: Los autores

La primera observación que resalta es que en Santa Elena no hay producción de huevos provenientes de gallinas criadas en planteles avícolas, lo cual coincide con el hecho de que no hay crianza de gallinas ponedoras. Por otro lado, dado que existe una clara correspondencia entre la producción de huevos y el número de gallinas, obviamente la producción de huevos es relativamente muy baja en esta provincia y la totalidad es para autoconsumo.

En resumen, con una producción pecuaria tan limitada, la provincia no está en capacidad de proveer la creciente demanda de productos alimenticios de origen pecuario.

\section{Conclusiones}

Si se acepta que, para ser parte de un sistema globalizado, es necesario contar con unos elementos (recursos tangibles e intangibles), estrategias (herramientas y tecnología), que permitan la mejora continua y el cambio permanente de manera proactiva; entonces es indispensable una planificación flexible y adecuada a las exigencias del sistema globalizado y de los estándares de calidad.

Así también, una de las formas para mejorar la administración de los programas y políticas gubernamentales e institucionales, es determinar y aplicar métodos que permitan evaluar de manera objetiva qué realmente sucede o puede suceder con los recursos naturales y humanos que son parte constitutiva del Estado, cómo se dinamizan estos recursos, cuáles aspectos deben superarse, incorporarse o reforzarse para proseguir con la tarea de brindar seguridad alimentaria a la ciudadanía.

El argumento atenuante para la provincia de Santa Elena, es que la búsqueda de un estado de bienestar de la sociedad peninsular no figuró como la premisa fundamental para la creación de la provincia; lo cual ha sido determinante para la Burgos, B/ Suárez, O. existencia de carencias y limitaciones de recursos propios; es decir, sin capacidad suficiente para generar auto gestión sustentable. Situación que mantendrá atada a la provincia a depender en alto grado de las asignaciones presupuestales del Estado como ya lo son otras provincias en la actualidad.

La provincia de Santa Elena posee ocho cultivos predominantes de acuerdo con la metodología implementada en la encuesta de superficie, producción y rendimiento agropecuaria continua (ESPAC) realizada por el Instituto Nacional de Estadística y Censos (INEC) en el 2015: banano, cacao, café, maracuyá, plátano, maíz duro choclo, maíz duro seco y soya.

De estos productos, el banano, el cacao, el café y el maracuyá no contribuyen de manera directa en la alimentación local pues están destinados principalmente a la exportación; la soya está destinada a la agroindustria para la elaboración de aceites y de igual manera el maíz duro para la elaboración de balanceados, por lo tanto, tampoco son de consumo directo sino indirecto. En definitiva, se puede decir que la contribución de Santa Elena a la alimentación diaria, está únicamente dada por el plátano. No se cuentan con datos de cuánto de estos productos se quedan en la península y cuánto se vende afuera, sin embargo, considerando que es relativamente poco lo que se produce, se puede argumentar que la provincia saca muy poco provecho de la siembra de estos productos.

De todo lo expuesto, se puede deducir que la producción agroalimentaria de la provincia de Santa Elena no es autosuficiente; en efecto la alimentación de la población peninsular depende ampliamente de productos importados de otras provincias; esto sitúa a la provincia en una situación vulnerable en cuanto a la soberanía y seguridad alimentarias que garanticen un crecimiento sano y adecuado de su población. 


\section{Recomendaciones}

La novel provincia de Santa Elena, a través de sus diferentes estamentos públicos y privados, necesita sentar las bases de una estructura productiva sólida que reduzca la dependencia y fomente la soberanía alimentaria.

Las autoridades técnicas vinculadas al agro deben trabajar muy de cerca con el sector privado sea este empresarial o comunitario sobre bases de colaboración mutua y transferencia efectiva de tecnologías adecuadas al medio. Concomitantemente, la inversión tanto pública como privada es clave para crear una infraestructura productiva eficiente y deben darse las facilidades para hacer atractivas a las mismas, ya lo han demostrado algunos desarrollos agroindustriales puntuales con buenos resultados en cuanto a rendimientos, es hora de un esfuerzo mancomunado para que estos proyectos se multipliquen y se extiendan en la geografía santaelenense.

En este nexo público-privado, el rol de la academia es fundamental para lograr las sinergias necesarias, promover la investigación en el campo de la producción agroalimentaria que sustente la planificación pública-privada y generar conocimientos y tecnologías relevantes al medio.

El fomento de la agroproducción es una ruta insustituible para el desarrollo sostenible a largo plazo y como tal debe ser el punto de partida de todo conglomerado que pretende convertirse en un referente en el contexto nacional y regional.

\section{Referencias bibliográficas}

[1] Constitución de la República del Ecuador (2008). Capítulo tercero Soberanía alimentaria, Art. 281.

[2] FAO (s.f.). El espectro de la malnutrición. http://www.fao.org/worldfoodsummit/span ish/fsheets/malnutrition.pdf

[3] Foro por la Soberanía Alimentaria (2007). Declaración de Nyéléni. Sélingué, Mali. http://nyeleni.org/spip.php?article291

[4] Freire W.B., Ramírez-Luzuriaga M.J., Belmont P., Mendieta M.J., Silva-Jaramillo M.K., Romero N., Sáenz K., Piñeiros P., Gómez L.F., Monge R. (2014). Tomo I: Encuesta Nacional de Salud y Nutrición de la población ecuatoriana de cero a 59 años. ENSANUT-ECU 2012. Ministerio de Salud Pública/Instituto Nacional de Estadísticas y Censos. Quito-Ecuador.

[5] Instituto Nacional de Estadística y Censos (INEC). Proyecciones Poblacionales. http://www.ecuadorencifras.gob.ec/proyec ciones-poblacionales/

[6] Instituto Nacional de Estadísticas y Censos. Encuesta de Superficie y Producción Agropecuaria Continua - ESPAC. http://www.ecuadorencifras.gob.ec/estadist icas-agropecuarias-2/ 